\title{
The Research and Development of Appropriate Metal Fittings for Drop- out Fuse Connecting Terminal
}

\author{
Li Bo, Hai Tianshu, Wang Qinghao, Pang Yanjun, Zhang Ning, Su Yang, \\ Yang Rui, Han Lin \\ Fushun Power Supply Company, Liaoning Electric Power Company Limited, State Grid, China, \\ fushunpowersupply@163.com
}

Keywords: drop-out fuse; cross bus; contact overheating; contact area; appropriate metal fitting

\begin{abstract}
. his paper discusses the problems existing in the use of drop-out fuse: due to wind swing and other reasons, the bonding point of $\mathrm{Cu}-\mathrm{Al}$ contact is easy to break, which may lead to the malfunction; the actual contact area between under contact and $\mathrm{Cu}-\mathrm{Al}$ contact in transformer lead wire is insufficient ,it is easy to cause overheating and burning of contact; the type of insulated conductor in transformer cross bus is general $35 \mathrm{~mm}^{2} \mathrm{JKLYJ}$, and cannot be installed ground, the distance between drop-out fuse upper contact and under contact is $0.3 \mathrm{~m}$, it is less than $0.7 \mathrm{~m}$, This does not meet the requirements of the installation of grounding wire. This paper not only introduced the design ideas and advantages of appropriate metal fitting, but also introduced the field usage. The appropriate metal fitting is simple to install, easy to operate, and other characteristics, it can greatly improve the power grid safety, economy and reliability.
\end{abstract}

\section{Introduction}

$10 \mathrm{kV}$ drop-out fuse can be installed in the transformer high-voltage side transformer, transformer and capacitor and line connections, provide overload and short circuit protection, can also be installed in the long line ends or branch lines, provide protection to the scope of protection. The drop-out fuse has simple structure, cheap price, convenient maintenance, small volume, and it is widely used in the distribution network. Its working principle is: fuse through the fusion tube, twisted at both ends, when normal, the contact between fusion tube upper moving contact and under static contact is reliable by fuse tension, When fault and fuse, the fuse breaks and forms the arc, weld tubes produced a large number of gas, the arc formed blowing arc, arc stretched and extinguish, and loss the fuse pull, under the action of gravity, the fuse pipe is falling down, cut off the circuit, forming obvious cut-off distance[1-3].

Drop-out fuse is made up of current-carrying part、 fuse tube、Insulating part and fixed part. The fuse tube includes: fuse, fuse, cap, operation ring on the moving contact, short axis. The fuse material is generally copper silver alloy, it has high melting point, and has a certain mechanical strength. At the top of fuse tube there is a release pressure cap, placing a fuse piece with low melting point. When high current, upper cap of the thin metal sheet melt formed double exhaust end; when switching on and off the small current, the thin metal sheet of lower cap melt and formed double ended exhaust; when the small current, the thin metal sheet of upper cap does not move, forming single ended exhaust[4].

\section{The existing problem of drop-out fuse}

Due to the economy, flexibility and the ability to adapt to the environment, the drop-out fuse as over current protection switching equipment is widely used in the primary side of transformer platform and the cable platform, in the process of application, it shows some disadvantages because of the construction of the under contact, the specific circumstances are as follows:

1) Due to wind swing and other reasons, the bonding point of $\mathrm{Cu}-\mathrm{Al}$ contact is easy to break, which may lead to the malfunction

2) The actual contact area between under contact and $\mathrm{Cu}-\mathrm{Al}$ contact in transformer lead wire is 
insufficient, it is easy to cause overheating and burning of contact;

3) The type of insulated conductor in transformer cross bus is general $35 \mathrm{~mm}^{2} \mathrm{JKLYJ}$, and cannot be installed ground, the distance between drop-out fuse upper contact and under contact is $0.3 \mathrm{~m}$,it is less than $0.7 \mathrm{~m}$, This does not meet the requirements of the installation of grounding wire.

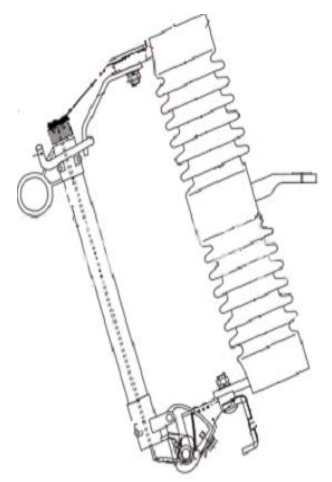

Fig.1 Structure diagram of drop-out

\section{The design scheme of appropriate metal fitting}

It develops a appropriate metal fitting for under contact connecting terminal in drop-out fuse, the material s election: $400 \mathrm{~mm}$ width; $30 \mathrm{~mm}$ thickness; $5 \mathrm{~mm}$ copper or aluminum tin row, it is installed in under contact of drop-out fuse. We make the terminal board downward extension of $150 \mathrm{~mm}$, and after doing a $105^{\circ}-115^{\circ}$ bending degree, horizontal extension $200 \mathrm{~mm}$, then make a $105^{\circ}-115^{\circ}$ bending degree at the end of the forward $50 \mathrm{~mm}$, leave a diameter of $6 \mathrm{~mm}$ galvanized screw to pass through the hole at the bus end of the forward $30 \mathrm{~mm}$, it is convenient to use the terminal to connect the terminal with the drop type terminal, and the connecting bolt should be protected. The equalizing file is used to polish the contact terminal of bus and drop-out under contact, and evenly coated conductive paste, increase the actual connection to the contact surface, to prevent the connection point of bad contact[5].

A $6 \mathrm{~mm}$ diameter hole is left at the second bending point of drop-out terminal row forward $20 \mathrm{~mm}$ which is used to install the bolted ground ring with protective pad for the grounding wire. By adjusting the angle of the bending angle of the terminal row effectively, above $0.7 \mathrm{~m}$ between the terminal board and the charged part of the upper contact is guaranteed. The drop-out terminal boards all make insulation treatment except $50 \mathrm{~mm}$ range at both ends
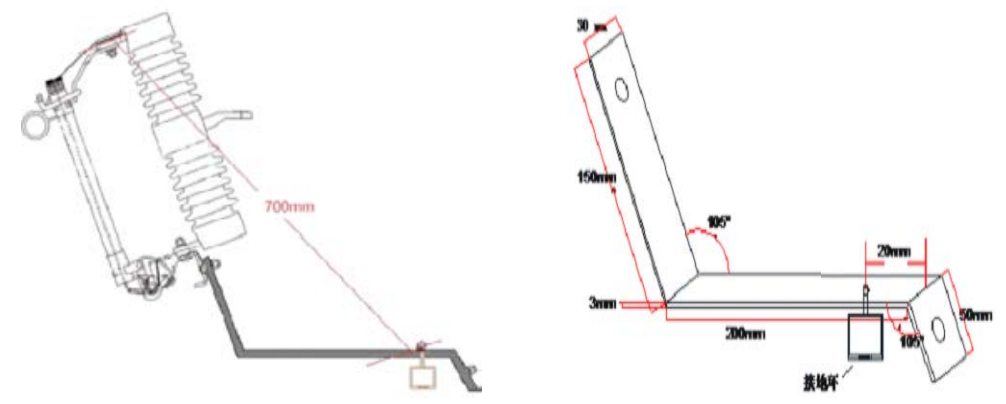

Fig.2 The appropriate metal fitting for under contact connecting terminal

\section{The effect and advantages of appropriate metal fitting}

The research and development of appropriate metal fitting for under contact connecting terminal in distribution platform drop-out fuse is successful in February 12, 2014, Figure 3 is actual photos, from Figure 3 it can be seen, appropriate metal fitting conform to the design requirements, fully meet the field use conditions. Its advantages are as follows:

1) It solves the problem that the bonding point of $\mathrm{Cu}-\mathrm{Al}$ contact is easy to break due to wind swing and other reasons 
2) It also finishes off the issue: the actual contact area between under contact and $\mathrm{Cu}-\mathrm{Al}$ contact in transformer lead wire is insufficient, it is easy to cause overheating and burning of contact;

3) It overcomes the trouble of fixing protective grounding wire safely and reasonably. The appropriate metal fitting has simple installation, simple operation, safe and practical, it can improve the safety, economy and reliability of power grid.

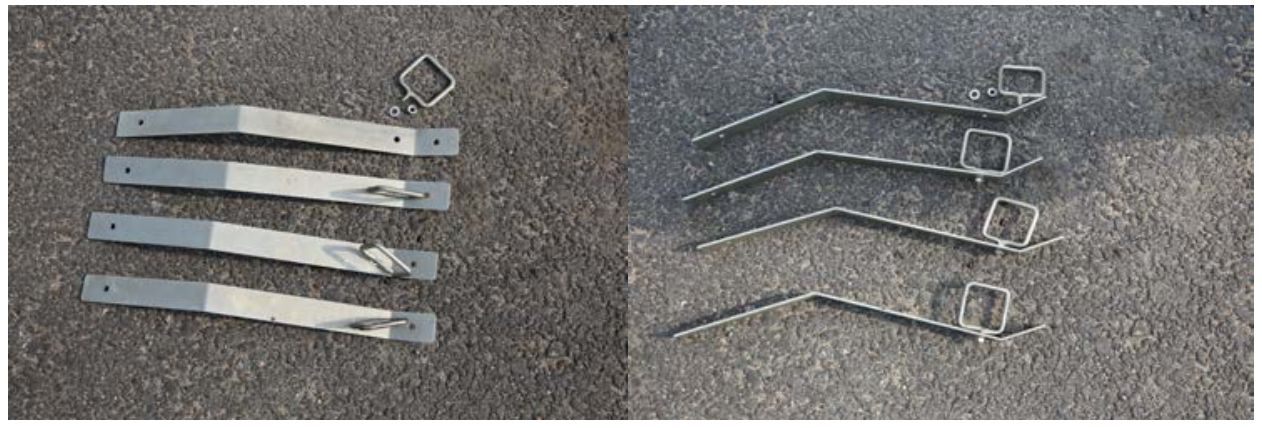

Fig.3 Actual photo of appropriate metal fitting

\section{Field application}

The NO.28 transformer of HeiHu line reformed in March 27, 2014, this appropriate metal fitting was installed at under contact. Six residential buildings on DongZhou area were blackout in May 14, 2014, maintenance personnel found that A phase of high-tension bushing connecting terminal on the NO.28 transformer of HeiHu line was burning. After work ticket, the working class began to work. The Safety measures: Open $10 \mathrm{kV}$ drop-out, hung the high voltage ground wire (DZ-01D10) on appropriate metal fitting for under contact connecting terminal .Then open the low-side switches and hung a group of the low voltage ground wire on the moving contact of secondary side isolator. The NO.28 transformer of HeiHu line restores power supply after maintenance for 30 minutes.

In the past, in order to ensure the safety of operators, the same type of fault needs to hang ground wire on the power supply side and load side. Because the high voltage ground wire could not be hung on $10 \mathrm{kV}$ under contact terminal, therefore, only to expand the scope of power outages, and hung the ground wire on the high voltage lead wire. The expansion of the scope of power outages is not only to apply to dispatching center, but also notice the important users within the scope of influence, it often appear that the electricity could not repair without power cut and ground wire. It not only expands the range of power, still unable to determine the time to repair. Through appropriate metal fitting for under contact connecting terminal, effectively solve ground wire hanging problem, avoid expanding the scope of power cut. Under the premise of ensuring safety, rapid repair and restore the power supply.
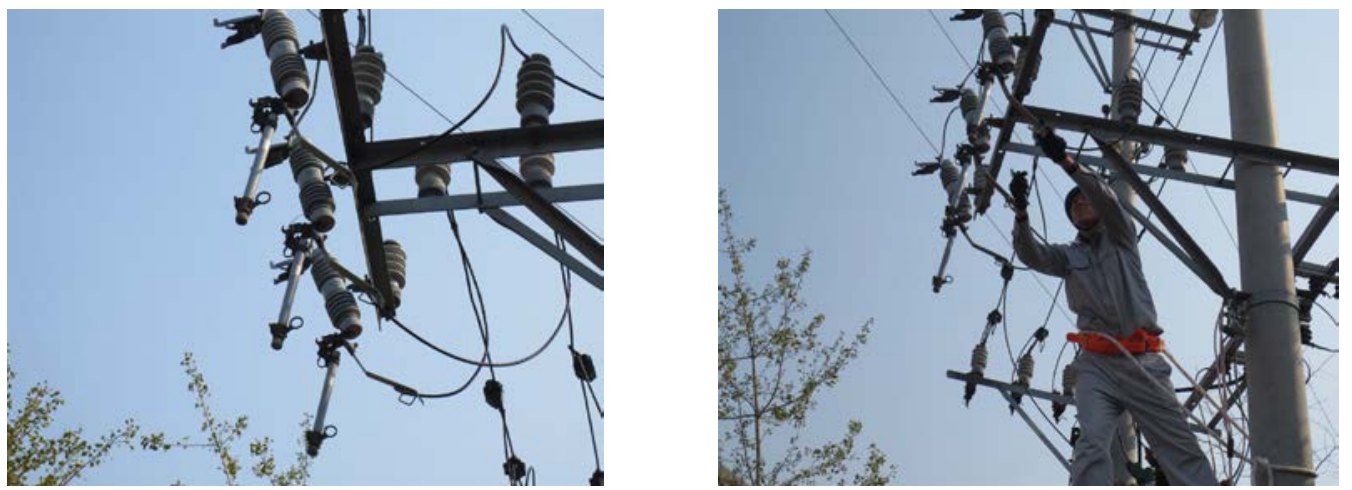

Fig.4 Field application of appropriate metal fitting 


\section{Conclusion}

In nearly a year's time, appropriate metal fitting for under contact connecting terminal in distribution platform drop-out fuse is widely used in the field, it is simple installation, convenient operation and so on ,not only shorten the operation time, and greatly improves the network safety, economy and reliability, has been widely used in Fushun power supply company.

\section{References}

[1] Fang Daqian. Quick-speed manual count transformer. China Water Power Press, 2004

[2] Chen Huagang, Preventive Test Method and Diagnosis Technology of Electric power Equipment. Science and Technology Press, 2001

[3] Chen Jiabin, Grounding Technique and Earthing Device. China Power Press, 2010

[4] Wang Changyu, Grounding Technology Security. China Water Power Press, 2008

[5] Zhang Xiaohui. Electrical Testing (Skills Training). China Water Power Press, 2010 